\title{
Maternal hospital deaths: clinical and sociodemographic characteristics in Yucatán, México
}

\section{Summary}

Objective: Describe the clinical and sociodemographic characteristics of maternal deaths that occurred in a hospital in Yucatán, Mexico, as well as propose recommendations to improve the care of pregnant women in order to help reduce in-hospital maternal mortality.

Material and methods: Retrospective, cross-sectional study conducted in a second-level care concentration hospital. The hospital database of maternal deaths that occurred during the 2014-2018 period was reviewed, as well as clinical summaries, maternal death reports, maternal death ratification forms, and hospital records.

Results: There were 54 maternal deaths, the year with the highest percentage was 2018 with $15(27.8 \%)$ deaths. Most of the women resided in municipalities with low and very low degrees of marginalization 23 (42.6\%); 48 (88.9\%) were engaged in housework; 13 had a history of previous abortion $(24.1 \%)$, with prenatal control $18(33.3 \%)$, used some contraceptive method $13(24.1 \%)$; and $21(38.9 \%)$ presented some complication during pregnancy. Direct maternal deaths were mainly hypertensive disorders with 9 (32.1\%) deaths. Indirect maternal deaths were mainly due to heart disease and arteriovenous disorders 6 (30.0\%). The Hospital Mortality Committee issued 98 (55.1\%) recommendations to improve emergency care, of which $32(32.6 \%)$ were related to the training of health personnel in the first level of care; and $22(22.5 \%)$ for the second level of care.

Conclusion: It is important to improve the care of the pregnant woman through the permanent training of health personnel to detect warning signs in the prenatal consultation and care of the obstetric emergency, in order to fully detect the problems that arise in the care chain of patients. Likewise, the training and updating of medical personnel for the correct completion of the basic causes of maternal death according to IDC-10 will be necessary for the correct certification of deaths.

Keywords: hospital maternal death, mortality committee
Volume II Issue 6 - 2020

\author{
Rodríguez Angulo Elsa María,' Uc Santos \\ Guillermo, ${ }^{2}$ Gómez Carro Salvador ${ }^{3}$ \\ 'Regional Research Center "Dr. Hideyo Noguchi ",Autonomous \\ University of Yucatan, México \\ ${ }^{2} \mathrm{~S}$ chool of Medicine, Autonomous University of Yucatan, México \\ ${ }^{3}$ General Hospital “Dr.Agustín O'Horán ”. Health Secretary, \\ México
}

Correspondence: Elsa María Rodríguez Angulo, Center for Regional Research "Dr. Hideyo Noguchi” from the Autonomous University of Yucatán, Avenida Itzáes, No. $490 \times$ Calle 59, Col. Centro, CP 97000, Mérida,Yucatán, México, Tel (999) 923-6I20 Ext. II2I,Email rangulo@mail.uady.mx

Received: December 23, 2020 | Published: December 29, 2020

\section{Introduction}

Maternal deaths are unfortunate events that predominate mainly in developing countries. A maternal death is defined as the death of a woman during pregnancy or within 42 days after its term, regardless of its duration or location. Maternal deaths are classified as direct obstetric, which are the result of complications of pregnancy (pregnancy, childbirth and the puerperium); and indirect obstetric, derived from existing diseases prior to pregnancy, or that appear during pregnancy, but which were not due to direct obstetric causes, but were aggravated by the physiological effects of pregnancy. Late maternal deaths are also defined, due to direct or indirect causes that occur after the puerperium, but not after one year. ${ }^{1}$

Maternal mortality continues to be a problem of a medico-social nature worldwide. The World Health Organization (WHO) estimates that 830 women die daily from complications related to pregnancy or childbirth, most of which can be attributed to 3 main preventable causes: hemorrhage, septicemia and hypertension in pregnancy. ${ }^{2,3}$ In addition, maternal mortality is considered as a parameter that reflects the degree of social development of a population, as well as the gaps in access and quality of health services.

Mexico is among the nations with medium-low maternal mortality, reporting in 2019 a total of 690 maternal deaths from direct and indirect obstetric causes; ${ }^{4}$ figure that has had a slow decline over the last ten years with marked heterogeneity within the country, variability over time that requires attention and resources within the nation. ${ }^{5}$
The biological and social determinants related to maternal deaths are multiple, such as maternal complications, poverty, inequality due to lack of opportunities for education and access to health and gender conditions, among others. These tend to adapt to the sociocultural variations of the different population groups, so that their identification and timely differentiation allow an adjustment to health programs to contribute to the reduction of mortality figures. The knowledge and technologies that exist today have not been sufficient to prevent maternal deaths. This study describes the clinical and sociodemographic characteristics of maternal deaths that occurred in a concentration hospital in Yucatán, México.

\section{Material and methods}

A cross-sectional, retrospective study carried out in a concentration hospital of the second level of care in the State of Yucatán, Mexico. From August to December 2019, the hospital database of maternal deaths was reviewed, as well as clinical summaries, maternal death reports, death ratification forms and hospital records of the Hospital Committee for Maternal and Perinatal Mortality of maternal deaths that occurred during the period 2014-2018. All deaths registered and judged as maternal deaths from direct and indirect obstetric causes, as well as late and non-obstetric ones, were selected.

The study was approved by the Hospital's Ethics and Research Committee with number CEI-2019-0033. For the certification of the cause of maternal death, the WHO Guide for the application of the International Classification of Diseases in its tenth revision (IDC- 
10) was used .1 In IDC-10 deaths have a causal relationship and/or temporary, are typified and defined as direct or indirect causes, deaths during pregnancy, childbirth and the puerperium, late maternal deaths and non-obstetric deaths. For each death, the recommendations made by the Hospital Committee on Maternal and Perinatal Mortality were reviewed and organized, which were classified by groups according to their basic causes.

The sociodemographic variables collected were age, place of residence, degree of marginalization, ${ }^{6,7}$ date of death, marital status, occupation, schooling and religion. The clinical variables were personal pathological history, number of pregnancies and abortions, use of contraceptives, presence of complications during pregnancy, prenatal care and type of maternal death.

Descriptive analysis was carried out in the Epi info program version 6.01 . With a $95 \%$ confidence level, considering significant values of $\mathrm{p}<0.05$, associations were calculated through the X2 test with Fisher's correction.

\section{Results}

During the study period, 54 maternal deaths were registered in the hospital database, the highest percentage occurred in 2018 with 15 $(27.8 \%)$ deaths. The mean age was 26 years and the age group with the highest number of maternal deaths was between 20 and 34years with $39(72.2 \%)$ deaths. Regarding the place of residence, the highest percentage of cases corresponded to women from municipalities with low and very low degrees of marginalization 23 (42.6\%); and municipalities with a high degree of marginalization 22 (40.7\%); Most of them lived in a free union $33(61.1 \%)$, dedicated themselves mainly to housework $48(88.9 \%)$, with basic education studies completed 30 $(55.5 \%)$ and with some religion $45(83.3 \%)$ (Table 1).

Table I Sociodemographic variables hospital maternal deaths 2014-2018

\begin{tabular}{lll}
\hline & Frequency & Percentage \\
\hline Year of death & & \\
2014 & 13 & 24.1 \\
2015 & 10 & 18.5 \\
2016 & 9 & 16.7 \\
2017 & 7 & 12.9 \\
2018 & 15 & 27.8 \\
& $\mathrm{n}=54$ &
\end{tabular}

\section{Age groups}

$\begin{array}{lll}15-19 \text { years } & 6 & 11.1 \\ 20-34 \text { years } & 39 & 72.2 \\ 35 \text { years or older } & 9 & 16.7 \\ & n=54 & \end{array}$

Degree of marginalization of the municipality of residence

\begin{tabular}{lll} 
Very low - Low & 2.3 & 42.6 \\
Means, medium & 9 & 16.7 \\
High & 22 & 40.7 \\
& $\mathrm{n}=54$ & \\
Civil status & & \\
Free Union & 33 & 61.1 \\
Married & 19 & 35.2 \\
\hline Single & 2 & 3.7 \\
& $\mathrm{n}=54$ & \\
\hline
\end{tabular}

Table continued...

\begin{tabular}{lll}
\hline & Frequency & Percentage \\
\hline Occupation & & \\
Housewife & 48 & 88.9 \\
Employee & 4 & 7.4 \\
Merchant & 2 & 3.7 \\
Scholarship & $\mathrm{n}=54$ & \\
Basic and more & 30 & 55.5 \\
Basic incomplete & 24 & 44.5 \\
& $\mathrm{n}=54$ & \\
Religion & & \\
Yes & 45 & 83.3 \\
No & 9 & 16.7 \\
& $\mathrm{n}=54$ & \\
\hline
\end{tabular}

When analyzing the clinical variables, it was found that $26(48.1 \%)$ women had some personal pathological history and the majority died during their second or more pregnancies $33(61.1 \%)$; with previous abortion 13 (24.1\%); and 18 (33.3\%) completed their prenatal care; 13 (24.1\%) used contraception and 21 (38.9\%) had complications during pregnancy (Table 2).

Table 2 Clinical variables of maternal deaths 2014-2018

\begin{tabular}{|c|c|c|}
\hline & Frequency & Percentage \\
\hline \multicolumn{3}{|c|}{ Pathological personal history } \\
\hline Yes & 26 & 48.1 \\
\hline No & 28 & 51.9 \\
\hline \multicolumn{3}{|l|}{ Gesta } \\
\hline 1 & 21 & 38.9 \\
\hline 2 or more & 33 & 61.1 \\
\hline \multicolumn{3}{|c|}{ Previous abortions } \\
\hline Yes & 13 & 24.1 \\
\hline No & 41 & 75.9 \\
\hline \multicolumn{3}{|c|}{ Prenatal control } \\
\hline Yes & 18 & 33.3 \\
\hline No & 36 & 66.7 \\
\hline \multicolumn{3}{|c|}{ Use of contraceptives } \\
\hline Yes & 13 & 24.1 \\
\hline No & 41 & 75.9 \\
\hline \multicolumn{3}{|c|}{ Complications during pregnancy } \\
\hline Yes & 21 & 38.9 \\
\hline No & 33 & 61.1 \\
\hline
\end{tabular}

Of the 54 maternal deaths, the majority were classified as direct obstetric deaths, with 28 (51.9\%) cases (Table 3).

Table 3 Classification of maternal death according to IDC-10

\begin{tabular}{lll}
\hline Death type & Number & Percentage \\
\hline Direct & 28 & 51.9 \\
Undirect & 18 & 33.3 \\
Non-obstetric & 7 & 12.9 \\
Late & 1 & 1.9 \\
Total & 54 & 100 \\
\hline
\end{tabular}


The complications that originated direct maternal deaths and that were located in the death certificate as the basic cause, were mainly hypertensive disorders during pregnancy, childbirth and the puerperium such as pre-eclampsia, eclampsia and HELLP syndrome with $9(32.1 \%)$ deaths; and obstetric hemorrhage, due to uterine atony and premature detachment of the normal inserted placenta with $7(25.0 \%)$ deaths. The main causes of indirect maternal deaths were heart disease and arteriovenous disorders with $6(33.3 \%)$ deaths; and neoplasms and autoimmune disorders, with $4(22.2 \%)$ deaths each (Table 4).

When comparing the number of direct and indirect maternal deaths, no significant differences were found $(2=0.0067 ; \mathrm{p}=0.9348)$. Similarly, having or not having prenatal care and dying was independent $(2=0.0931 ; p=0.7603)$. When analyzing maternal deaths by degree of marginalization, significance was obtained both for women who came from municipalities with a low and very low degree of marginalization $(2=8.70 ; \mathrm{p}=0.0031)$, and for women who came from municipalities with a high degree $(2=7.65 ; \mathrm{p}=0.0056)$ (Table 5$)$.

The recommendations made by the Hospital Committee on
Maternal and Perinatal Mortality were 98 and they were distributed into 7 groups (Table 6). The most frequent were aimed at training first and second level health personnel, with 32 (32.7\%) and 22 $(22.4 \%)$ recommendations, respectively. For the first level personnel, the importance of training for a correct identification, classification and management of normal-evolutionary and high-risk pregnancies was emphasized; and for the second level, the recommendations emphasized adequate compliance with the protocols for obstetric emergencies, as well as the importance of submitting the necessary cases to the Ethics Committee for evaluation. Other recommendations were the need to provide supplies and material resources and medical equipment to the different care units of obstetric emergencies, including transfer units (14.3\%); as well as recommendations to form an effective communication network between the entities involved in the care of patients in serious conditions at the state and interstate level $(12.2 \%)$; and the strengthening of the efficiency of in-hospital services that are involved in the indirect care of critically ill patients, such as the blood bank and clinical laboratory (7.2\%). Recommendations were also given for the optimal follow-up of each case of maternal death, in order to provide feedback and improve health services $(6.1 \%)$.

Table 4 Basic causes of maternal death by type. IDC- I0. $n=54 *$

\begin{tabular}{llll}
\hline Type & Cause & No. & Percentage \\
\hline \multirow{2}{*}{ Direct } & Hypertensive disorders in pregnancy, childbirth and the puerperium & 9 & 32.1 \\
& Obstetric hemorrhage & 7 & 25 \\
& Genitourinary infections & 4 & 14.3 \\
& Pregnancy ending in abortion & 2 & 7.2 \\
& Other obstetric complications & 6 & 21.4 \\
Total & & 28 & 100 \\
& Heart disease and arteriovenous disorders & 6 & 33.3 \\
Hint & Neoplasies & 4 & 22.2 \\
& Autoimmune disorders & 4 & 22.2 \\
& Gastrointestinal conditions & 4 & 16.7 \\
Total & Respiratory Conditions & 3 & 1 \\
\end{tabular}

*Late maternal deaths I (I.8\%); and non-obstetric 7 (I3.0\%)

Table 5 Analysis of maternal deaths by degree of marginalization

\begin{tabular}{llll}
\hline & Number and percentage & $\mathbf{X}^{2}$ & P value \\
\hline Low and very low grade vs. medium grade & $23(72 \%)$ vs. 9 (28\%) & 8.7 & 0.0031 \\
High grade vs. medium grade & $22(70 \%)$ vs. 9 (30\%) & 7.65 & 0.0056 \\
Low and very low grade vs. high grade & $23(51 \%)$ vs. 22 (29\%) & 0.04 & 0.8452 \\
\hline
\end{tabular}

Table 6 Recommendations of the hospital committee on maternal and perinatal mortality

\begin{tabular}{lcc}
\hline Recommendations & Number & Percentage \\
\hline I. Train first and second level care personnel in obstetric emergency & 54 & 55.1 \\
2. Provide supplies and equipment for obstetric emergencies & 14 & 14.3 \\
3. Implement an effective inter-institutional communication network & 12 & 12.2 \\
4. Streamline the service of blood and blood products in the hospital & 7 & 7.2 \\
5. Follow up on cases of maternal death & 6 & 6.1 \\
6. Supervise and integrate the private health sector & 5.1 & 98 \\
Total
\end{tabular}




\section{Discussion}

The present study made it possible to describe the current panorama of maternal mortality registered in a concentration hospital in the State of Yucatan, Mexico, to which pregnant women from different municipalities of the State and neighbors were referred. Although there was apparently a downward trend in the percentage of deaths during the first four years studied, the last year presented a higher percentage of mortality than the first year. Therefore, it could not be said that the problem has been controlled, since there are still spikes in mortality at less than five years of follow-up.

In the literature, it is mentioned that the periods of greatest risk of maternal complications are those found at the extremes of childbearing age, that is, in those over 35 and under 14years of age. ${ }^{8,9}$ However, women In our study, young people died, in the group with the highest fertility in reproductive life, as has been reported in local studies of maternal mortality of Yucatecan women. ${ }^{10}$

The risk that the place of residence represents with cases of maternal death is of varied opinion, on whether or not it influences mortality. Pisanty-Alatorre5, highlighted a heterogeneity regarding marginalization in the country, finding regions with high marginalization rates that presented low maternal mortality and vice versa. In the women in this study, the prevalences of mortality according to the degree of marginality of their municipalities of residence were similar. Mérida, the capital of the State, stands out among the municipalities with a very low degree of marginalization, with the largest number of population, which would be expected that due to the level of development in this urban environment there would be fewer maternal deaths. It will be important to locate the areas where the women resided in the state capital, to delimit a map that provides guidance on the existence of an area with lack of access to health services. It would also be important to address whether it is only this factor that is influencing the lack of survival or there are added factors that must be addressed to reduce deaths in the city. It has been observed in local studies that despite the fact that in urban areas there are hospitals to provide immediate response to the care of complications, there is inequality in the population, especially in the conurbation areas of the capital, which are part of the so-called belt of poverty. Freyemerth Enciso, carried out an analysis of the social determinants of maternal mortality in Mexico, highlighting the need to guarantee access to health services for the entire population,

Likewise, it is also known that the biological aspect has a substantial influence on the genesis, development and outcome of various pathologies; such as personal history of diseases, conditions or factors that can alert health personnel in prenatal care. In the women in this study, we found that in almost half of the patients (48.14\%) at least one of them was present, such as the previous cesarean section, whose scar is considered a risk factor for other conditions such as ectopic pregnancy. which in turn is referred to as the main cause of maternal death in the first trimester of pregnancy. ${ }^{11,12}$

Other factors found were multiparity and uterine curettage due to previous abortions, which are considered the main risk factors for presenting a hemorrhagic event during pregnancy, childbirth and postpartum $;{ }^{13}$ so its knowledge in this study was important for the treating personnel, who must be prepared for any eventuality and avoid complications that compromise the life of the mother and fetus. Good control and management of preconception risk through contraceptive methods is a determining element in the reduction of general morbidity and mortality in the female population and the real possibility of reducing or avoiding the damage that, from the biopsychosocial and environmental, a mother or her child may suffer. ${ }^{14}$ In our study, we were able to observe that this control was only present in $24.07 \%$ of the deceased women. Therefore, efforts should be redoubled to promote family planning methods in the population, as another important element that women can have to prevent pregnancy and maternal-fetal risks.

Maternal and child health in Mexico is followed, in part, through prenatal control, being of utmost importance in the prevention of maternal deaths, because health personnel can comprehensively address the patient through a file or card perinatal, promoting that lowrisk pregnancies receive at least 5 prenatal consultations, at determined gestational ages, in order to detect maternal-fetal risks in a timely manner. ${ }^{15}$ According to the established guidelines, prenatal control should begin in the first few 8 weeks of gestation. However, pregnant women frequently come to their control when they consider that they may be pregnant (in rural areas it occurs when midwives tell them), covering long periods between conception and the first consultation. In our study, more than half of the women who died (66.6\%) did not receive prenatal care. So it would be necessary to study what are the factors that influence not attending your consultations. ${ }^{16,17}$

Therefore, continuous training of medical personnel at all levels is necessary to achieve quality care. Mejía C et al. ${ }^{18}$ Acknowledge that the most feasible measure to get women to go to prenatal care is to ensure their empowerment, essentially in areas of limited accessibility, highlighting the importance of recognizing warning signs, with the aim of reducing the barriers that influence the delay of attention and referral. ${ }^{18}$

In the present study, the most frequent obstetric complications that caused deaths could be identified, highlighting hypertensive disorders and obstetric hemorrhage as the main causes of direct maternal death; data that agree with other reports in Mexico, ${ }^{18}$ which stimulate the reinforcement and acquisition of new strategies to address these complications. However, recently with the arrival of the SARs-CoV2 virus in Mexico, the cases of death from COVID-19 in pregnant women are occupying the first place as a cause of maternal death, displacing hemorrhage and hypertensive disorders; a very important challenge that the health system will face in the future. ${ }^{19}$

With regard to maternal deaths from heart disease, greater attention should be paid to the control of pregnancy in women with heart disease; And even before pregnancy, counseling will be very important in these women who are at high risk of complications. This same condition has been presented in other countries as the main cause of indirect maternal death. ${ }^{20}$ Another data that drew attention was the number of non-obstetric events, that is, those that did not derive from pregnancy as a cause of death, but that exceeded the frequency of the first indirect cause; and that they were mainly due to infectious processes related to the lung and liver. Hence the importance that these conditions can be detected in time during prenatal control, which, although they do not derive from pregnancy.

Errors in the completion of the death certificate and its coding have been previously reported on a larger scale, through the intentional search and reclassification of maternal deaths in the country. ${ }^{21}$ In Yucatan, failures in the completion of certificates have also been reported that contributed to the underreporting, ${ }^{22}$ there are no recent studies that show that these failures have been corrected. Therefore, the correct classification is necessary, since it offers valuable information on maternal deaths that occur in the hospital, and in this way to be able to propose and implement measures that can be carried out at all levels of care to prevent true causes why women are dying. Hence 
the importance of highlighting the work of the Hospital Maternal and Perinatal Mortality Committee in charge of rectifying or ratifying the diagnoses of the causes of death, of which there is sometimes very little documented information; and identify the limitations that existed in the chain of care for the patient, from the first level of care to their arrival at the hospital; and in this way it was possible to issue recommendations to contribute to the prevention of maternal deaths due to complications. ${ }^{23}$

The Committee's recommendations include permanent training at all levels on obstetric complications, how to prevent them and their treatment; This same recommendation has also been given by specialists in order to guarantee adherence and follow-up to protocols and standards on prenatal care, to identify high-risk pregnancies in a timely manner and to channel them in time for more specialized care; emphasizing the importance of training staff at the first level of care, where the cases with complications come from. Villanueva Egan et al. Refer to this level, pointing out that the most effective interventions are those that provide continuous care from infancy and extend through pregnancy, childbirth and the puerperium; Therefore, quality care must be reassessed and offered, thereby generating patient adherence and achieving a healthy pregnancy. ${ }^{24}$ The second level of care also received numerous recommendations, requiring constant training of health personnel in emergency care obstetrics, ensuring adequate monitoring of the standards established for the care of each case. The need to purchase equipment and drugs from both the transfer units and the hospital units, which could be used in case of obstetric emergencies, was also highlighted. Demanding the constant training of health personnel in obstetric emergency care, ensuring adequate monitoring of the standards established for the care of each case.

In Yucatán, the delays in the care of the patient with obstetric complication, since the complication begins, its transfer and its care in the hospital, have also been reported by specialists in obstetric emergencies, who have given recommendations that coincide with those given the Committee in this study. ${ }^{17}$ To reduce these delays, the Committee recommended the importance of ensuring interinstitutional communication in the referral of patients, in order to guarantee their transfer in adequate conditions, with the necessary support measures until the patient reaches the hospital; and in turn prepare the personnel who will receive the case, reducing the time of delay in the attention that is vital. Other inpatient services that support the obstetric area with seriously ill patients are.

\section{Conclusion}

Hospital maternal deaths can be prevented if quality care is performed during prenatal control from the first 8 weeks of gestation in order to prevent complications that can prevent death; recognizing in time the warning signs and symptoms that indicate that the patient is getting complicated and being able to refer the case to a specialized level. The health services must coordinate with other institutions and sectors to guarantee a good quality of care from the locality, its transfer and care when arriving at the hospital. With all the analysis, a call is made to the union of the health services involved in each case of maternal death, in order to fully detect the problems that arise in the chain of care for patients, as well as to issue and request the measures that can prevent more cases. This involves both the public and private sectors, which also had recommendations from the Committee to analyze their facilities and care protocols that they carry out to achieve the decisive management of high-risk obstetric patients. Training in obstetric emergencies at all levels is a main recommendation to contribute to the reduction of maternal deaths.
Similarly, it is important to empower medical personnel to correctly fill in the basic causes of maternal death according to IDC10 , to guide maternal health policies towards programs that propose strategies aimed at preventing the causes for which they are dying. Prematurely pregnant women.

\section{Acknowledgments}

None.

\section{Funding}

None.

\section{Conflicts of interest}

The authors declare that there are no conflicts of interest.

\section{Thanks}

We are grateful to the Hospital that provided the facilities for access to the corresponding documents.

\section{References}

1. WHO guide for the application of IDC-10 to deaths during pregnancy, childbirth and the puerperium: IDC-MM. World Health Organization; 2012.

2. QUIEN. Maternal mortality. 2019.

3. Global strategy for women's, children's and adolescent health (20162030). All the women, all the children. 2015.

4. Maternal mortality observatory in Mexico. Weekly epidemiological surveillance report. 2020.

5. Pisanty Alatorre Julio. Inequities in maternal mortality in Mexico: an analysis of inequality at the sub-state level. Public Health of Mexico. 2017;59(6):639-649.

6. National Population Council. Marginalization Index by state and municipality 2015. Mexico; 2015.

7. National Institute of Statistic and Geography. Total maternal deaths. Information from 1990 to 2017. Tabulation by year of registration, entity and site of occurrence. Mexico.

8. Vera PG Claudio, Carvajal Cabrera Jorge A. Severe maternal morbidity and near losses at the extremes of reproductive age: results of a national multicenter cross-sectional study. Rev Chil Obstet Ginecol. 2014;79(6):554-557.

9. Soni Trinidad C, Gutiérrez Mateos A, Santa Rosa Moreno FJ, et al. Maternal morbidity and mortality and risk factors associated with an obstetric emergency. Gynecology and Obstetrics of Mexico. 2015;83(02):96-103.

10. Angulo EMR, Andueza-Pech MG, Peña YO. Characteristics of maternal death among Mayan Women in Yucatan, Mexico. In: Schwartz D, editors. Maternal Death and Pregnancy-Related Morbidity Among Indigenous Women of Mexico and Central America. 2018:249-270.

11. Freyermuth Encino Graciela. Social determinants of maternal mortality in Mexico. CONAMED magazine. 2016;21(01):25-32.

12. Escobar Padilla B, Pérez López CA, Martínez Puon H. Risk factors and clinical characteristics of ectopic pregnancy. Rev Med Inst Seguro Soc. 2017;55(03):278-285.

13. Solari A, Solari C, Wash A, et al. Postpartum hemorrhage. Main etiologies, their prevention, diagnosis and treatment. Rev Med Clin Counts. 2014;25(6):993-1003. 
14. González Portales Alicia, Rodríguez Cabrera Aida, Jiménez Ricardo Maybelis. Preconception risk and its link with maternal health. Rev Cubana Med Gen Integr. 2016;32(3).

15. Official Mexican standard NOM-007-SSA2-1993. Care of women during pregnancy, childbirth and the puerperium and of the newborn. Criteria and procedures for the provision of the service.

16. Gonzaga Soriano M, Zonana Nacach M, Anzaldo Campos M, et al. Prenatal care and hospital maternal mortality in Tijuana, Baja California. Public Health of Mexico. 2014;56(01):32-39.

17. Rodríguez-Angulo E, Pech GA, Vázquez RZ. Global risk of death due to delays in the care of pregnant women with obstetric complication in yucatan, Mexico. Obstet Gynecol Int J. 2018;9(2):118-122.

18. Mejía C, Cárdenas M, Miñan M, et al. Reference time associated with the cause of maternal mortality in the Regional Maternal and Child Teaching Hospital "El Carmen", 2009-2015. Rev Chil Osbtet Ginecol. 2018;83(1):6-14.

19. Health. Health Secretary. General Directorate of Epidemiology. Weekly report of immediate notification of maternal death. Week 50 of 2020.
20. Geetha R Menon, Lucky Singh, Palak Sharma, et al. National Burden Estimates of healthy life lost in India, 2017: an analysis using direct mortality data and indirect disability data. Lancet Glob Health. 2019; 7:e1675-1684.

21. Torres Luis Manuel, Rhenals Ana Luisa, Jiménez Aline, et al. Intentional search and reclassification of maternal deaths in Mexico: the effect on the distribution of causes. Public Health of Mexico. 2014;56(04):333347 .

22. Rodríguez-Angulo Elsa, Andueza-Pech Guadalupe, MonteroCervantes Landy, et al. Under-registration of maternal deaths in Mayan communities of eastern Yucatán. Revista Biomédica. 2009;20(2):90-98.

23. Aguli Ruíz- Rosas, R, Cruz- Cruz, PdR. Causes of maternal mortality in the Mexican Institute of Social Security, period 2009-2012. Revista Médica del Instituto Mexicano del Seguro Social. 2014;52(4):389-396.

24. Villanueva Egan L, Shiavon Ermani R. Evidence-based Latin American interventions to reduce maternal mortality. CONAMED magazine. 2013; 18(01):21-30. 